\title{
A Novel Combined Approach for Metastatic Breast Cancer with Dural and Leptomeningeal Disease with an Impressive Clinical Outcome: A Case Study
}

\author{
Julie Taguchi, Christopher Duma, M. A. Nezami \\ Pacific Medical Center of Hope, Fresno, USA \\ Email:amnezami@yahoo.com
}

How to cite this paper: Taguchi, J., Duma, C. and Nezami, M.A. (2018) A Novel Combined Approach for Metastatic Breast Cancer with Dural and Leptomeningeal Disease with an Impressive Clinical Outcome: A Case Study. Journal of Cancer Therapy, 9 , 274-280.

https://doi.org/10.4236/jct.2018.93025

Received: February 7, 2018

Accepted: March 16, 2018

Published: March 19, 2018

Copyright $\odot 2018$ by authors and Scientific Research Publishing Inc. This work is licensed under the Creative Commons Attribution International License (CC BY 4.0).

http://creativecommons.org/licenses/by/4.0/

\begin{abstract}
Concurrent dural and leptomeningeal metastatic carcinomatosis are very rare and have a poor prognosis. Here we present a woman with advanced estrogen receptor (ER) positive and progesterone receptor (PR) positive breast cancer who presented with leptomeningeal disease. Patient underwent multi targeted epigenetic therapies applied in a protocol called MTET. She continued to respond to the interval treatment, which consisted only of the nutraceutical agents. Here we discuss her case in detail and we believe that such an example might be applied to other patients in this situation resulting clinical improvement and less toxicity.
\end{abstract}

\section{Keywords}

Breast Cancer, Metastatic, Leptomeningeal Disease, Dural Disease

\section{Introduction}

Concurrent dural and leptomeningeal metastatic carcinomatosis are very rare and have a poor prognosis. In the literature, advanced breast carcinomas, bone-to-dura spread has been hypothesized to arise from dissemination of tumor cells from the bone, especially the vertebral bodies, with retrograde reflux of tumor cells into veins, venousplexi, and subsequently the dura. Conventional intrathecal infusions of cytotoxic agents have failed to improve survival in majority of patients. Data from the Cleveland Clinic, Humboldt University in Berlin, and Karolinska Hospital in Stockholm have shown a median survival ranging from 4.2 to 6.5 months despite radiation therapy (RT) to the brain. 
If the tumor is small in size (less than $3 \mathrm{~cm}$ ), Gamma knife stereotactic radiosurgery (GKRS) is an option. Gammaknife survival rates are similar to surgery plus whole brain radiation or whole brain radiation alone and are generally ineffective. The majority of patients progress despite systemic therapy and die in less than 6 months [1]-[7].

Here we present a woman with advanced estrogen receptor (ER) positive and progesterone receptor (PR) positive breast cancer who presented with left facial paralysis, numbness to the chin and scalp, double vision, and severe headaches, then diagnosed with metastatic duraland leptomeningeal disease with possible intraparenchymal disease and involvement of the cavernous sinus, cranial fossa and microinvasion to brain parenchyma by the tumor.

\section{Methods}

The patient underwent combined systemic therapy by receiving a patented series of neutroceutical infusions consisting of called MTET (multi-targeted epigenetic therapy), along with GKRS, and minimal oral chemotherapy. She experienced a surprising overall improved physical response and radiological findings; all together it created a meaningful clinical response to the minimally toxic treatment. With the MTET alone, she continued to respond to the interval treatment, which consisted only of the neutroceutical agents.

\section{Discussion}

Here we discuss her case in detail and we believe that such an example might be applied to other patients in this situation resulting clinical improvement and less toxicity.

\section{Case Study}

A forty-seven year-old woman with an initial diagnosis of stage 2A ER-positive, PR-weak positive, HER2 negative multifocal ductal carcinomas of the left breast declined adjuvant tamoxifen and RT after a left lumpectomy and negative sentinel node biopsy. The OnctoypeDx Recurrence Scores of both tumors were 27 and 33. This patient used alternative therapies including GcMAF, an injectable immune modulator with dietonly to discover 5 months later with increasing hip pain, she harbored extensive bony metastases on her PET scan. The bony lesions were diffuse and destructive in all areas involving the thoracic and lumbar spine. She required a left hip replacement.

The patient accepted taking $10 \mathrm{mg}$ of Tamoxifen with monthly injections of Denosumab (XGEVA) andlater stopped the Denosumab due to the side effects of prolonged bone pain and reactions. Her oncologist referred her for evaluation and therapy since the patient wished to avoid cytotoxic chemotherapy. The patient's main complaint was pain and inability to walk due to progressive disease in the bones. Her initial labs 7/12/16 prior to the therapy showed very high tumor marker levels (CA 15.3, 27.29 and CEA), elevated LDH and alkaline phos- 
phatase, and positive circulating tumor cell DNA (c tDNA) with Guardant 360 testing for PI3K and FGFR1. The serum showed abnormally high HER2 positivity. Thrombocytopenia (platelets $=71,000$ ) and calcium of 12.4 were assumed due to extensive bony metastases.

Treatments and results: She started IV epigenetic therapies with the MTET protocol, which she received on daily basis for two weeks, then tapered to twice a week. MTET protocol consists of patented combination of polyphenols formulated to modify the histone deacetylases along with DNA demethylating effect.

The patient reported improved quality of life after the very first day of treatment and continued to improve clinically. Her ECOG score improved from 3 to 1 after two weeks. Labs repeated after two weeks on 7/26/16 showed a marked reduction (20 percent) in all her tumor markers (See chart). Her c tDNA became undetectable. Calcium normalized and the serum Her 2 level dropped from 124 to 66 without anti-HER2 agents, measured 3 weeks later.

Targeted therapies (trastuzumab, everolimus $2.5 \mathrm{mg}$ ) and hormonal blockade (GNRH antagonist monthly) were added to MTET epigenetic therapies after $7 / 26 / 16$. Such combination showed improved results 2 weeks later. Her tumor markers decreased again CA 27.29 dropped from 1136 to 804 measured on 8/5 and 8/16/16; CEA changed from 2237 to 1620; CA 15.3 dropped down from 954 to 757; LDH fell from 925 to 556; alkaline phosphatase reduced from 162 to 125 (please see Table 1).

Table 1. Trend of Tumor markers.

\begin{tabular}{|c|c|c|c|c|c|c|c|c|c|c|c|c|}
\hline & 2016 & $\begin{array}{l}7 / 12 / 16 \\
\text { baseline }\end{array}$ & $\begin{array}{l}7 / 26 / 16 \\
\text { week } 3\end{array}$ & $\begin{array}{l}8 / 5 / 16 \\
\text { week } 4\end{array}$ & $\begin{array}{l}8 / 16 / 16 \\
\text { week } 5\end{array}$ & $\begin{array}{l}8 / 23 / 16 \\
\text { week } 6\end{array}$ & $\begin{array}{l}10 / 16 \\
\text { mo } 3\end{array}$ & $\begin{array}{c}\text { 11/16 } \\
\text { mo } 4 \\
\text { Hospital }\end{array}$ & $\begin{array}{l}1 / 17 \\
\text { mo } 6\end{array}$ & $\begin{array}{l}3 / 17 \\
\text { mo } 8\end{array}$ & $\begin{array}{l}3 / 27 \\
\text { mo } 8\end{array}$ & $\begin{array}{r}4 / 6 / 17 \\
\text { mo } 9\end{array}$ \\
\hline CA 15-3 & $\mathrm{NA}$ & 1055 & 875 & 945 & 757 & 895 & & & 962 & $\mathrm{NA}$ & & 201.2 \\
\hline CA-2729 & & 1192 & 1000 & 1136 & 804 & 952 & 3436 & & 1114 & & 209 & 187.2 \\
\hline CEA & & 2707 & 2237 & 2368 & 1620 & 1852 & & & 635 & & & 235.7 \\
\hline LDH & & 545 & 925 & 742 & 556 & 685 & & & 291 & & & 141 \\
\hline AlkPhos & & 184 & 162 & 172 & 125 & 134 & & & 244 & & & 108 \\
\hline ctDNA & & Pos & Neg & $\mathrm{N} / \mathrm{A}$ & N/A & N/A & N/A & & $\mathrm{N} / \mathrm{A}$ & $\mathrm{N} / \mathrm{A}$ & & \\
\hline HER 2 & & 124 & N/A & 66 & N/A & 28 & & & 24 & $\mathrm{~N} / \mathrm{A}$ & & 23.3 \\
\hline Calcium & & 13.9 & 7.5 & 8.0 & 7.1 & 8.5 & & & 8.0 & & & 6.5 \\
\hline Platelet & & $109 \mathrm{~K}$ & $65 \mathrm{~K}$ & $90 \mathrm{~K}$ & $122 \mathrm{~K}$ & $160 \mathrm{~K}$ & & & $212 \mathrm{~K}$ & $313 \mathrm{~K}$ & & \\
\hline PET & $\mathrm{Xa}$ & & & & & $\mathrm{Xb}$ & & & & $\mathrm{Xc}$ & & \\
\hline $\begin{array}{c}\text { MRI } \\
\text { Brain }\end{array}$ & $\mathrm{Xd}$ & & & & & & $\begin{array}{c}\mathrm{Xe} \\
\text { GKRS }\end{array}$ & & $\mathrm{Xf}$ & $\mathrm{Xg}$ & & \\
\hline MTET & & $\mathrm{X}$ & $\mathrm{X}$ & $\mathrm{X}$ & $\mathrm{X}$ & $\mathrm{X}$ & $\mathrm{X}$ & - & $\mathrm{X}$ & $\mathrm{X}$ & & \\
\hline
\end{tabular}

Xa: PET diffuse mets to bone; Xb: Significant reduction in SUV activity of nearly all boney mets. Xc: PET neg FDG; diffuse blastic changes in bone; low attenuation liver lesions-neg FDG. Xd: MRI brainbaseline-left paramedial enhancement. No intracranial mets; skull mets. Xe: MRI brain; diffuse dural metastatic disease, involving superior sagittal sinus, left cavernous, and right temporal ovale, with possible right temporal and left parietal microinvasion. Xf: MRI brain-reduction in duralmass. Xg: MRI brain-continued reduction of dural mass. MTET: Multi-targeted epigenetic therapy. 
The serum Her 2 level continued to drop to 28 from 124.1 at baseline (please see Figure 1).

On 4/6/17 while patient has been off ANY conventional therapies for over 6 months, her labs showed significant improvement on MTET therapy. Her CA 27.29 dropped down to 187, her CA 15.3 dropped down to 201, her LDH down

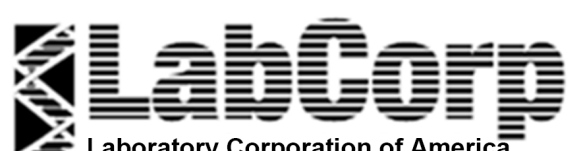

Laboratory Corporation of America

Serial Monitoring Report

1912 Alexander Drive, Research Triangle Park. NC 27709 (919) 361-7700

Report Date: 04/12/2017

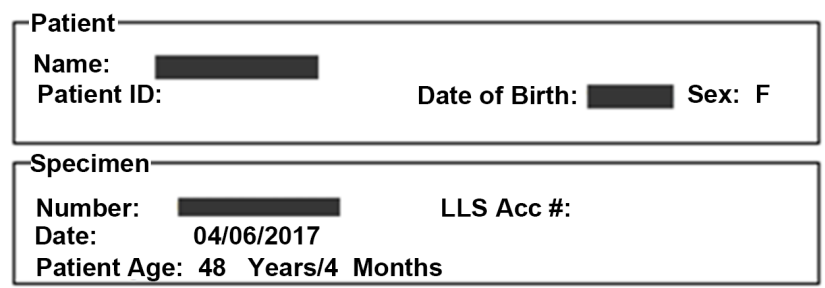

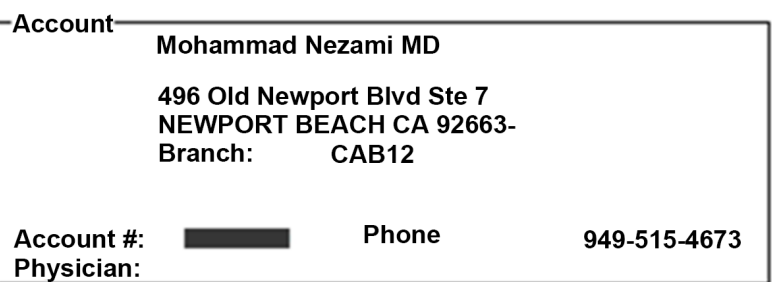

O Her2/Neu Quan

$\mathrm{ng} / \mathrm{mL}$

$$
100.0 \%
$$

$$
124.1
$$

90.

$80.0-$

$60.0-\oint$

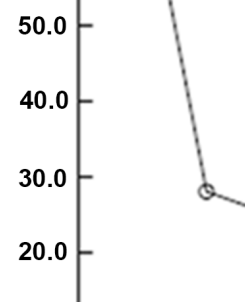

10.0
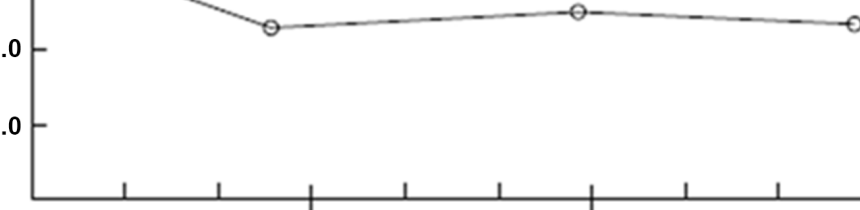

X-Axis: MONTH

Oct 16

Jul 17

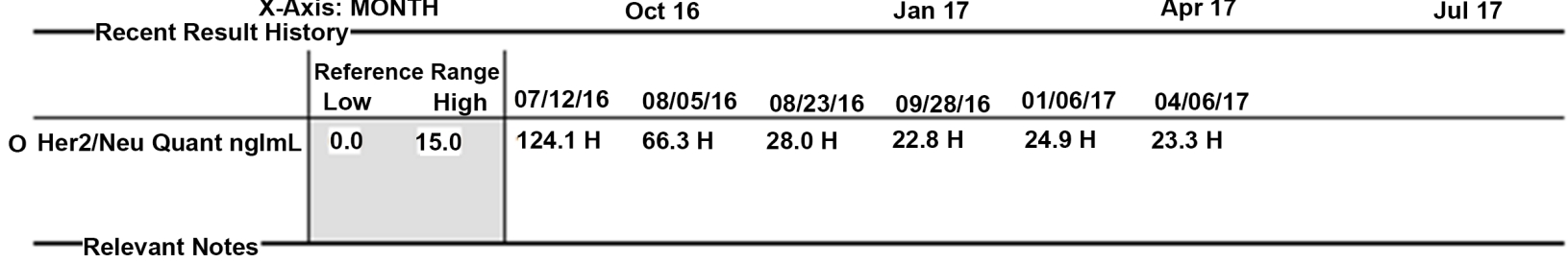

Andre Valcour. PhD. DABCC. FACB. Director of Esoteric Immunoassay Arundhati Chatterjee. MD. Medical Director

Figure 1. Serum Her2 level. 
to 141, her ALK-p down to 108, CEA down to 235, serum Her2 level at 23.3, Calcium at 6.5 .

Restaging PET scan 8/2016 showed significant response to interval therapy including a near impressive reduction of her bony metastases with decreased sizes and SUV activity on all lesions. (Please see Image 1).

A brain MRI 10/19/16 evaluated a right facial paralysis and numbness involving the left side of her face and scalp, in addition to complaints of progressive double vision. The MRI confirmed the presence of diffuse dural metastatic disease, involving superior sagittal sinus, left cavernous, and right temporal ovale, with possible right temporal and left parietal microinvasion of the brain. Lumbar puncture was negative for cytology. The patient was referred to the neurosurgery department at Hoag Hospital for evaluation and possible treatment.

Patient was offered whole brain radiation in addition to intrathecal chemotherapy, which she refused. It was decided that patient was a candidate for GKRS if the leptomeningeal and intraparenchymal disease could be possibly treated with systemic therapy. The patient accepted this combined approach understanding of the potential low response considering the extent of her disease and the site. She decided to hold her chemotherapy and targeted therapies all together in November 2016, and proceeded with maintenance epigenetic therapies. Recovering from viral and bacterial pneumonia in November, she did not receive any conventional treatments 4 weeks prior to the follow up MRI other than a dose of denosumab. She recovered her neurological status very quickly with minimal physical deficitsandresidual numbness of her chin. This included resolution of the left facial paralysis and double vision.

Repeat MRI of the brain on $1 / 7 / 17$, showed reduction of her tumor by 50 percent in the volumetric size from $1.463 \mathrm{~cm}^{3}$ to $0.731 \mathrm{~cm}^{3}$ or diameter changed from $16.5 \times 6.84 \mathrm{~mm}$ to $11.8 \times 6.68 \mathrm{~mm}$. Repeat MRI March 2017 showed moreimprovement from $8 \times 3.8 \mathrm{~cm}$ to $5 \times 2.8 \mathrm{~cm}$ in dural thickness and persistence
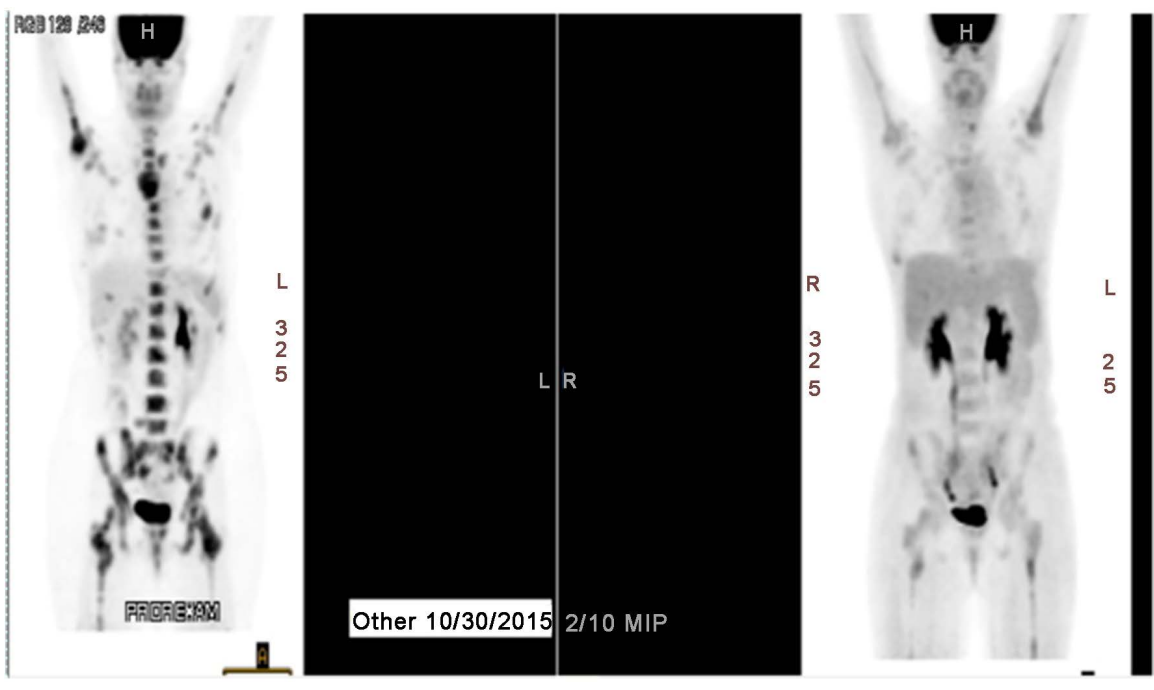

Image 1. PET scan. 
Waring: Images for multiple studies and/or patients printed
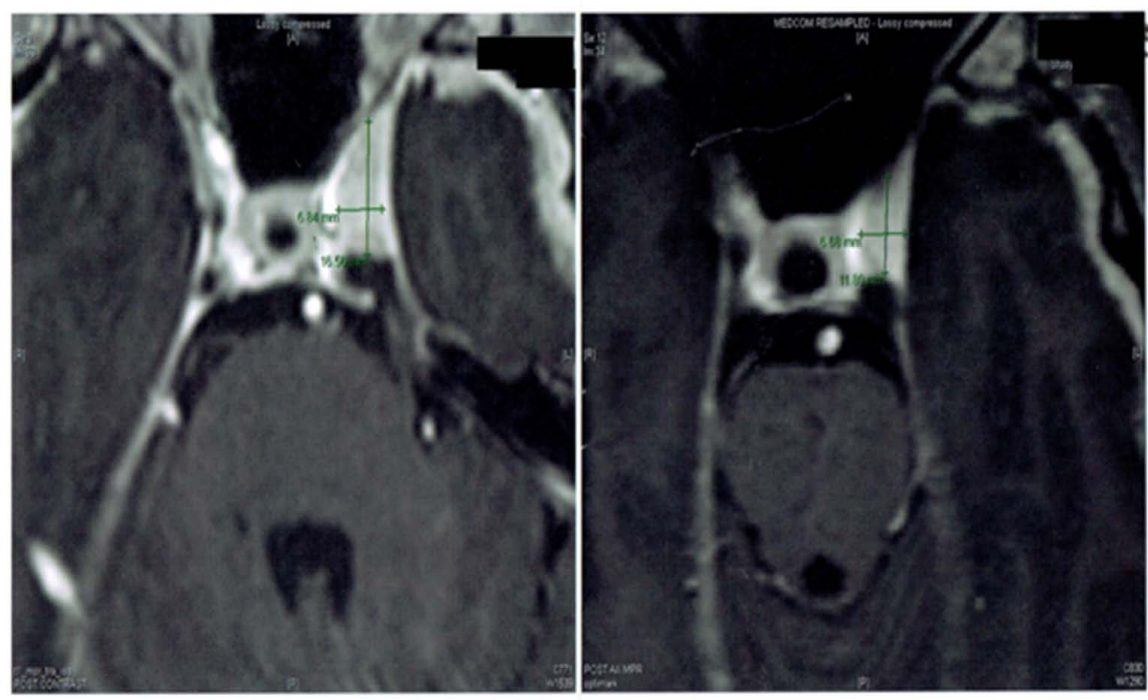

Image 2. Brain MRI.

of disease through to the right foramen ovale (Please see Image 2).

Labs drawn at the same time as the MRI confirmed her systemic response 5 months from baseline with improvement of tumor markers and LDH. Her CA 15.3 dropped from 2172 to 962 , her CA 27.29 dropped from 2049 to 1114, and CEA from 2025 to 635.

The patient remained on MTET treatment and continued to improve in all objective clinical measures. As of this writing, she had passed her expected survival. Such an amazing response in a patient with advanced dural and meningeal disease is remarkable and further requires attention and could potentially impact the current standard of care.

\section{Conclusion}

The magnitude of response in this patient appears better than expected to the current standard of care outcome. Further trials are needed to support application of this novel combinational approach in advanced metastatic and leptomeningeal metastatic disease as a potential candidate for application for orphan drug designation.

\section{References}

[1] Kakani, C., Preeshagul, I.R., Fisher, R., et al. (2015) Management of Isolated Leptomeningeal Metastasis as the Initial and Sole Site of Recurrence in a Patient with Early-Stage Breast Cancer. International Cancer Conference Journal, 4, 101. http://link.springer.com/article/10.1007/s13691-014-0178-2

[2] Chang, E.L. and Lo, S. (2003) Diagnosis and Management of Central Nervous System Metastases from Breast Cancer. The Oncologist, 8, 398-410. http://theoncologist.alphamedpress.org/content/8/5/398.long

[3] Mahmoud-Ahmed, A.S., Suh, J.H., Lee, S.Y., et al. (2002) Results of Whole Brain Radiotherapy in Patients with Brain Metastases from Breast Cancer: A Retrospec- 
tive Study. International Journal of Radiation Oncology * Biology * Physics, 54, 810-817. https://www.ncbi.nlm.nih.gov/pubmed/12377333

[4] Lentzsch, S., Reichardt, P., Weber, F., et al. (1999) Brain Metastases in Breast Cancer: Prognostic Factors and Management. European Journal of Cancer, 35, 580-585. https://www.ncbi.nlm.nih.gov/pubmed/10492631

[5] Fokstuen, T., Wilking, N., Rutqvist, L.E., et al. (2000) Radiation Therapy in the Management of Brain Metastases from Breast Cancer. Breast Cancer Research and Treatment, 62, 211-216. https://link.springer.com/article/10.1023/A:1006486423827

[6] Nayak, L., Abrey, L.E. and Iwamoto, F.M. (2009) Intracranial Dural Metastases. Cancer, 115. http://onlinelibrary.wiley.com/doi/10.1002/cncr.24203/pdf

[7] Rosner, D., Nemoto, T. and Lane, W.W. (1986) Chemotherapy Induces Regression of Brain Metastases in Breast Carcinoma Patients: Update Study. Cancer, 58, 832-839.

http://onlinelibrary.wiley.com/doi/10.1002/1097-0142(19860815)58:4\%3C832::AIDCNCR2820580404\%3E3.0.CO;2-W/full 